\title{
Optical anisotropy in self-assembled InP quantum dots
}

\author{
Mitsuru Sugisaki,* Hong-Wen Ren, and Selvakumar V. Nair \\ Single Quantum Dot Project, ERATO, JST, Tsukuba Research Consortium, 5-9-9 Tokodai, Tsukuba, Ibaraki 300-2635, Japan \\ Kenichi Nishi \\ Optoelectronics and High Frequency Device Research Laboratories, NEC Corporation, 34 Miyukigaoka, \\ Tsukuba, Ibaraki 305-8501, Japan \\ Shigeo Sugou \\ Single Quantum Dot Project, ERATO, JST, Tsukuba Research Consortium, 5-9-9 Tokodai, Tsukuba, Ibaraki 300-2635, Japan \\ and Optoelectronics and High Frequency Device Research Laboratories, NEC Corporation, 34 Miyukigaoka, \\ Tsukuba, Ibaraki 305-8501, Japan \\ Tsuyoshi Okuno \\ Institute of Physics, University of Tsukuba, Tsukuba, Ibaraki 305-8571, Japan \\ Yasuaki Masumoto \\ Single Quantum Dot Project, ERATO, JST, Tsukuba Research Consortium, 5-9-9 Tokodai, Tsukuba, Ibaraki 300-2635, Japan \\ and Institute of Physics, University of Tsukuba, Tsukuba, Ibaraki 305-8571, Japan
}

(Received 22 October 1998)

\begin{abstract}
Strong optical anisotropy is observed in the photoluminescence (PL) bands of both the InP self-assembled quantum dots and the $\mathrm{Ga}_{0.5} \mathrm{In}_{0.5} \mathrm{P}$ matrix. From the linearly polarized PL spectra measured under weak excitation, we found that large size quantum dots show strong anisotropy. The luminescence from a single quantum dot observed by the micro-PL technique revealed a doublet fine structure of the exciton levels that obey the linear polarization selection rule. The observed fine structure is shown to arise from an interplay of the electron-hole exchange interaction and the asymmetric crystal structure of the $\mathrm{InP} / \mathrm{Ga}_{0.5} \mathrm{In}_{0.5} \mathrm{P}$ system. [S0163-1829(99)50608-5]
\end{abstract}

Recently there has been strong interest in lowdimensional semiconductor structures such as quantum wells, wires, and boxes. From the basic physics viewpoint, an exciton confined in a zero-dimensional system is an attractive subject because of interesting physical properties reflecting dimensionality such as $\delta$-function-like density of states, enhanced oscillator strength, and strong optical nonlinearity. In the past few years, the self-assembled quantum dots (SAD's) have emerged as an attractive system for such studies. $^{1-3}$

Theoretically, the electronic structures of the InP (Ref. 4) and (Ga)InAs (Refs. 5-7) SAD's have been studied. A symmetric shape of the quantum dot is usually assumed to simplify the calculations. However, since the quantum dots grown under the Stranski-Krastanov mode are highly strained due to the lattice mismatch between the matrix and the SAD's, the energy levels of the confined exciton are influenced by the matrix in addition to the shape of the quantum dots. In real systems, therefore, the electronic levels of the confined excitons suffer perturbations due to strain and possible structural anisotropies of the matrix, and the electron states eventually become asymmetric. The asymmetry would manifest as energy splittings and optical anisotropy of the confined exciton states. In this paper, we report the optical anisotropy in the InP SAD's observed using conventional and microphotoluminescence systems, hereafter referred to as macro- and $\mu$-PL, respectively.
InP quantum dots embedded in $\mathrm{Ga}_{0.5} \mathrm{In}_{0.5} \mathrm{P}$ were grown on a Si-doped (001) oriented GaAs substrate by means of a gas-source molecular beam epitaxy. ${ }^{8}$ After an undoped $\mathrm{Ga}_{0.5} \mathrm{In}_{0.5} \mathrm{P}$ (lattice matched to the GaAs substrate) buffer layer $160 \mathrm{~nm}$ thick was grown on the substrate, the selfassembled InP islands were formed at $480^{\circ} \mathrm{C}$ (sample 1) or $520^{\circ} \mathrm{C}$ (sample 2 ) by deposition of nominally 4 monolayers InP. In order to investigate their optical properties, these islands were buried by a $\mathrm{Ga}_{0.5} \mathrm{In}_{0.5} \mathrm{P}$ layer $160 \mathrm{~nm}$ thick. The average size of the SAD's are estimated by cross-sectional transmission electron microscopy (TEM) (see Table I), and from atomic force microscopy, the size dispersions of the SAD's are estimated to be less $30 \%$. For photoluminescence (PL) measurements, an Ar-ion laser was used as the excitation light source. An edge of the sample was glued on the cold finger of the sample holder and the sample was cooled by cold helium gas in a flow-type cryostat. The PL was led to a 50-cm single monochromator using an optical fiber, and detected by a charge coupled device camera. The spectral resolution of this system is better than $0.3 \mathrm{meV}$. In order to measure the PL spectra from a single quantum dot, micropatterns with $10 \mu \mathrm{m} \times 10 \mu \mathrm{m}$ square were drawn on the sample by means of photolithography. After wet etching by $\mathrm{HCl}: \mathrm{H}_{2} \mathrm{O}=2: 1$ at $30{ }^{\circ} \mathrm{C}$, the mesa size was reduced to less than $4 \mu \mathrm{m} \times 4 \mu \mathrm{m}$. For the measurement of the polarization dependence of $\mu$-PL, a microscope objective lens was used to reduce the PL coming from outside of the mesa and a film polarizer was set inside the microscope. ${ }^{9}$ 
TABLE I. Growth temperature, size, and the optical properties of the InP SAD's in the $\mathrm{Ga}_{0.5} \operatorname{In}_{0.5} \mathrm{P}$ matrix. For comparison, those of $(\mathrm{Ga}) \mathrm{InAs} / \mathrm{GaAs}$ are also summarized.

\begin{tabular}{|c|c|c|c|c|}
\hline Sample No. & $\begin{array}{c}\text { Growth } \\
\text { temperature }\left({ }^{\circ} \mathrm{C}\right)\end{array}$ & $\begin{array}{l}\text { Size of SAD's } \\
\left(\mathrm{nm}^{3}\right)\end{array}$ & $\begin{array}{c}\text { Degree of } \\
\text { matrix }(\%)\end{array}$ & $\begin{array}{l}\text { arization in } \\
\text { SAD's }(\%)\end{array}$ \\
\hline InP (Sample 1) & 480 & $35 \times 45 \times 6$ & 83 & 44 \\
\hline InP (Sample 2) & 520 & $40 \times 48 \times 5$ & 40 & 13 \\
\hline $\mathrm{In}_{0.5} \mathrm{Ga}_{0.5} \mathrm{As}^{\mathrm{a}}$ & 520 & $17 \times 33 \times 3$ & & 16 \\
\hline InAs $s^{b}$ & 480 & $13 \times 15 \times 3$ & & 32 \\
\hline InAs on $(311) \mathrm{A} \mathrm{GaAs}^{\mathrm{c}}$ & 500 & & & 13 \\
\hline
\end{tabular}

${ }^{\mathrm{a} S e e}$ Ref. 11.

${ }^{\mathrm{b}}$ See Ref. 12.

${ }^{\mathrm{c}}$ See Ref. 13.

The solid and the dotted curves in Fig. 1(a) show the PL spectra at $4 \mathrm{~K}$ of sample 1 polarized along the [1 $\overline{1} 0]$ and [110] directions of the GaAs substrate, respectively. The PL bands observed at $1.52 \mathrm{eV}$ and $1.94 \mathrm{eV}$ arise from the GaAs substrate and the $\mathrm{Ga}_{0.5} \operatorname{In}_{0.5} \mathrm{P}$ matrix, respectively. The PL peak coming from the SAD's was observed at $1.67 \mathrm{eV}$. Even when the excitation power is decreased to 100 times weaker than this, the peak energy, the bandwidth, and the shape of this PL band are the same. Thus, the spectrum reflects the size distribution of the SAD's, and the luminescence arises from the radiative decay of the confined excitons in the ground state.

The PL peaks from the GaAs substrate do not show any polarization dependence as expected for cubic symmetry. The PL peaks from the $\mathrm{Ga}_{0.5} \mathrm{In}_{0.5} \mathrm{P}$ matrix and the $\mathrm{InP}$ SAD's, however, show clear polarization dependence, although both bulk $\mathrm{Ga}_{0.5} \mathrm{In}_{0.5} \mathrm{P}$ and bulk InP have the same symmetry as GaAs. The PL spectrum was not sensitive to the polarization of excitation under band-to-band excitation, im-

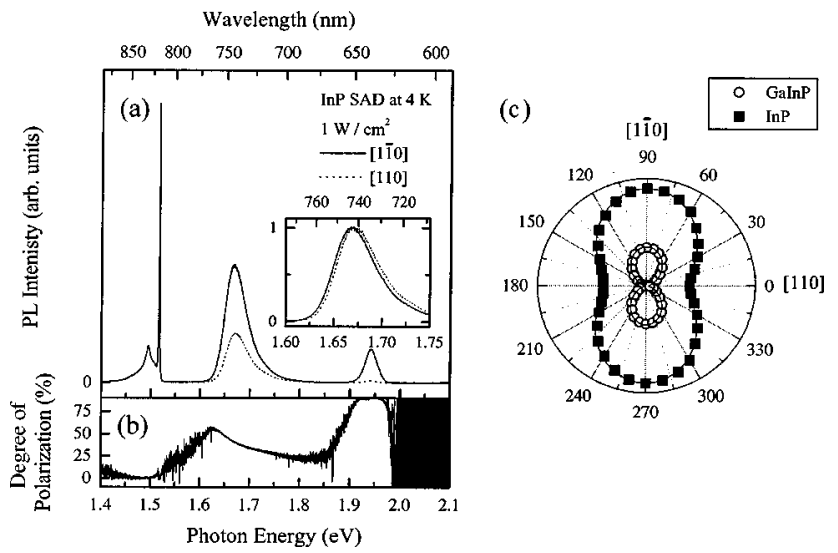

FIG. 1. (a) Polarization dependence of the PL spectra under weak excitation at $4 \mathrm{~K}$ observed for [1 $1 \overline{1} 0]$ (solid line) and [110] (dotted line) polarization. The polarization dependence appears in the PL bands of the InP SAD's and the $\mathrm{Ga}_{0.5} \operatorname{In}_{0.5} \mathrm{P}$ matrix. Inset: The normalized PL spectra of the InP SAD's bands. The energy separation of the peaks is $5 \mathrm{meV}$. (b) Degree of polarization of the PL spectra shown in Fig. 1(a). At the PL peaks of the InP SAD and the $\mathrm{Ga}_{0.5} \mathrm{In}_{0.5} \mathrm{P}$ matrix, it is $44 \%$ and $83 \%$, respectively. (c) Polar plots of the polarized PL peak intensities from $\mathrm{Ga}_{0.5} \mathrm{In}_{0.5} \mathrm{P}$ matrix (open circles) and the InP SAD's (closed squares). They show twofold symmetry, and can be fitted by Eq. (2). plying that the memory of polarization is lost before the carriers excited in $\mathrm{Ga}_{0.5} \mathrm{In}_{0.5} \mathrm{P}$ matrix relax into the quantum dots. The peak intensity of the luminescence band observed for [ $1 \overline{1} 0]$ polarization, which is parallel to the long axis of the SAD's, is more than twice as strong as that observed for [110] polarization.

The degree of linear polarization $P$ defined by

$$
P=\frac{I_{[1 \overline{10}]}-I_{[110]}}{I_{[1 \overline{1} 0]}+I_{[110]}}
$$

is plotted in Fig. 1(b), where $I_{[1 \overline{1} 0]}$ and $I_{[110]}$ are the PL intensities observed for the [1 $1 \overline{1} 0]$ and [110] polarizations, respectively. It has a peak at $1.63 \mathrm{eV}$, which is at the lower energy edge of the PL band from the InP SAD, and gradually decreases with increasing the photon energy. This suggests that large size quantum dots have stronger optical anisotropy than small ones. The degrees of polarization at PL peaks of the InP SAD's and the $\mathrm{Ga}_{0.5} \mathrm{In}_{0.5} \mathrm{P}$ matrix are $44 \%$ and $83 \%$, respectively.

The peak intensity of the PL bands of the InP SAD's and the $\mathrm{Ga}_{0.5} \mathrm{In}_{0.5} \mathrm{P}$ matrix are plotted in Fig. 1(c) as a function of the polarization angle of observation $\theta$. Both bands exhibit a twofold symmetry and have a maximum value for [1 10$]$ polarization. They are fitted by

$$
I=A \sin ^{2} \theta+B \cos ^{2} \theta
$$

where $A$ and $B$ correspond to the peak intensities of the PL bands observed for [1 10$]$ and [110] polarizations, respectively. This relation was found in the ordered $\mathrm{Ga}_{0.5} \operatorname{In}_{0.5} \mathrm{P}$ by Wei et al. ${ }^{10}$ As shown by the solid lines in Fig. 1(c), not only the PL from the $\mathrm{Ga}_{0.5} \mathrm{In}_{0.5} \mathrm{P}$ matrix but also that from the InP SAD's can be fitted by Eq. (2). This suggests that the InP SAD's have a twofold symmetric structure with symmetry axes along [110] and [1 10$]$ directions.

The inset of Fig. 1(a) shows the normalized PL spectra at the peaks of each InP SAD band. The PL peak observed for [1 10$]$ polarization is shifted to the lower energy side of that observed for [110] polarization by about $5 \mathrm{meV}$.

The polarization dependence of the PL spectra in (Ga)InAs SAD's has been earlier reported as summarized in Table I, and the anisotropy was attributed to the anisotropic 


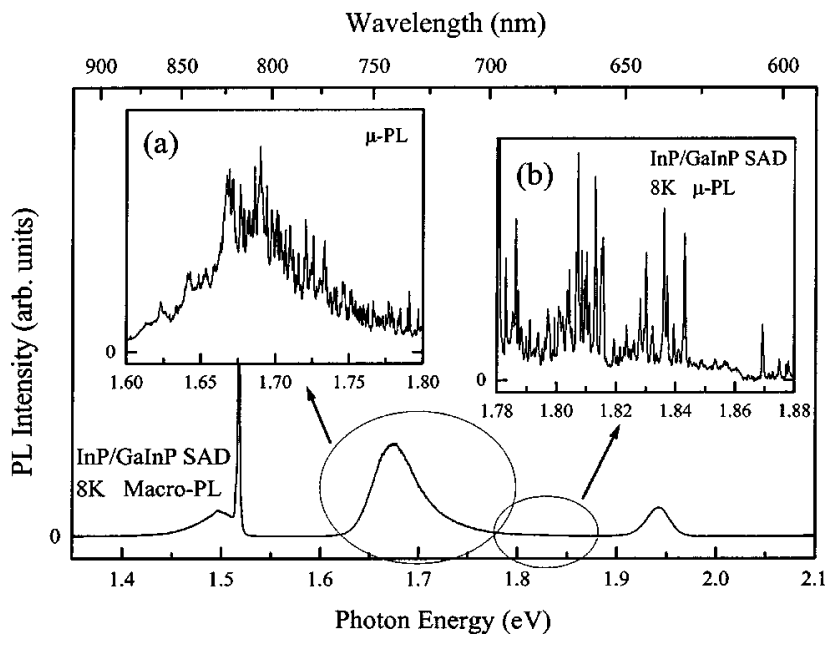

FIG. 2. Below: Macro-PL spectra at $8 \mathrm{~K}$. Insets (a) and (b): $\mu$-PL spectra of the mesa processed sample. Each $\delta$-function-like PL line arises from the radiative decay of the confined exciton.

shape of the SAD's. ${ }^{11-13}$ The optical anisotropy of sample 2, which was grown at a higher temperature than sample 1, was very weak in comparison with sample 1 , although the difference of the size and the shape of the SAD's between these samples is small (see Table I). Therefore, the observed strong optical anisotropy in sample 1 is not mainly due to the anisotropic shape of the SAD's. TEM study suggests that the optical anisotropy in the $\mathrm{Ga}_{0.5} \mathrm{In}_{0.5} \mathrm{P}$ matrix could be due to the formation of composition modulation planes along the [150] and [001] directions. ${ }^{8}$ We found that the degree of modulation in $\mathrm{Ga}_{0.5} \mathrm{In}_{0.5} \mathrm{P}$ grown at lower temperature is stronger, and then InP SAD's show the stronger optical anisotropy. In this case, since $\mathrm{Ga}_{0.5} \mathrm{In}_{0.5} \mathrm{P}$ matrix has $C_{2 \mathrm{v}}$ or lower symmetry, the optical anisotropy of the PL from $\mathrm{Ga}_{0.5} \mathrm{In}_{0.5} \mathrm{P}$ is observed. SAD's are thus embedded in an anisotropic matrix that would be subjected to an anisotropic strain. ${ }^{14}$ An anisotropic lattice structure of the SAD's is the most probable origin for their optical anisotropy. To clarify this further, we studied the luminescence from individual quantum dots by $\mu$-PL.

Figure 2 shows the comparison of the typical PL spectra of sample 1 at $8 \mathrm{~K}$ measured by means of the macro-PL system (the main spectrum of Fig. 2) and the $\mu$-PL system [insets (a) and (b)]. In the $\mu$-PL spectra, many sharp PL lines were observed at the higher energy side of the PL peak of the SAD's band, as shown in Fig. 2(a). Each PL peak corresponds to the radiative annihilation of the exciton confined in a single quantum dot and reflects $\delta$-function-like density of states. The envelopes of the PL spectra measured by means of macro- and $\mu$-PL system are almost the same. Thus, most of the sharp lines observed in $\mu$-PL are considered to arise from the ground state of confined excitons.

The $\mu$-PL measurements were also made for the energy range between the PL peak of the SAD's and the PL band of $\mathrm{Ga}_{0.5} \mathrm{In}_{0.5} \mathrm{P}$ matrix, as shown in Fig. 2(b). Although the macro-PL intensity is weak under weak excitation, the $\mu$-PL lines coming from single quantum dots were clearly observed. In the energy region shown in Fig. 2(b), the number of the PL lines is reduced because the areal density of the

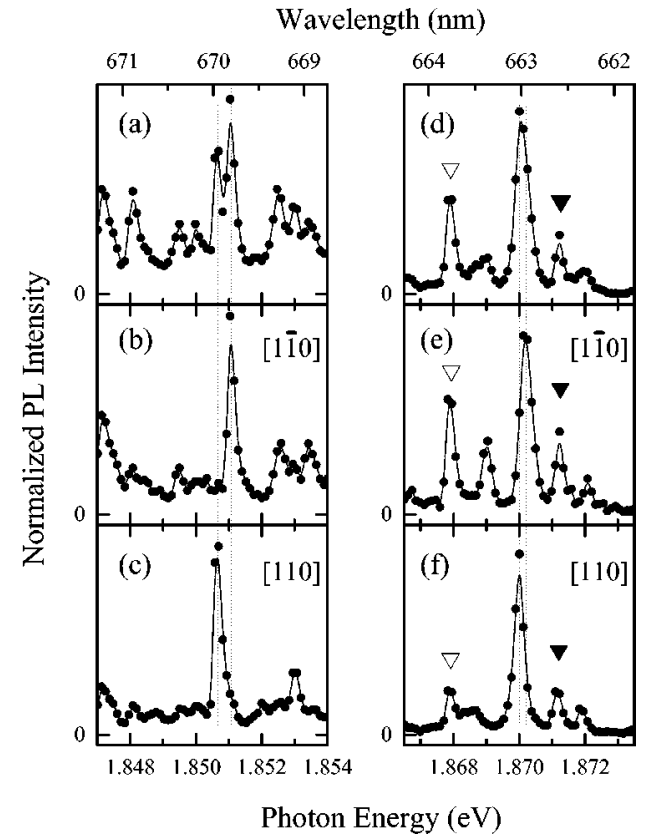

FIG. 3. Normalized $\mu$-PL spectra at $8 \mathrm{~K}$ obtained without [spectra (a) and (d)] and with polarizer along the [1 10$]$ [(b) and (e)] and [110] [(c) and (f)] directions. The solid lines are guides to the eyes. The separation of the broken lines are $400 \mu \mathrm{eV}$ (left) and $190 \mu \mathrm{eV}$ (right). Each spectrum is normalized at the peak.

small sized SAD's is low. Thus the polarization dependence of the PL from a single quantum dot was studied in this region.

The spectra (a) and (d) in Fig. 3 are observed without the polarizer, while the spectra (b) and (e) are observed for the [1 $1 \overline{1} 0]$ polarization, and (c) and (f) for the [110] polarization. The most important feature seen in the $\mu$-PL spectrum is the doublet nature of each peak. Each constituent of the doublet is fully polarized, i.e., one is along [110] and the other is along [ $1 \overline{1} 0]$. For example, a doublet with an energy separation of $\Delta E \simeq 400 \mu \mathrm{eV}$ at $1.851 \mathrm{eV}$ is clearly resolved in the polarized spectrum [Figs. 3(b) and 3(c)]. Theoretical analysis described below shows that the fine splitting arises from the electron-hole exchange interaction in the presence of structural asymmetry.

When the symmetry is lowered from $T_{\mathrm{d}}$ to $C_{2 \mathrm{v}}$, the single particle levels will lose all degeneracies except that due to spin. Thus, the hole levels would be twofold degenerate with the Bloch part of the wave functions, written in terms of the $X, Y$, and $Z$ valence-band orbitals of the original cubic material, given by

$$
\begin{gathered}
\left|h_{1}\right\rangle=\left(e^{i \phi}|X\rangle-i e^{-i \phi}|Y\rangle\right) \uparrow+c|Z\rangle \downarrow \\
\left|h_{2}\right\rangle=\left(e^{-i \phi}|X\rangle+i e^{i \phi}|Y\rangle\right) \downarrow-c|Z\rangle \uparrow,
\end{gathered}
$$

where $\phi$ and $c$ are constants. The electron levels are also twofold degenerate but $s$-like, and may be written as $\left|e_{1}\right\rangle$ $=|S\rangle \uparrow$ and $\left|e_{2}\right\rangle=|S\rangle \downarrow$. Consequently, the electron-hole pair states are fourfold degenerate unless the electron-hole exchange interaction is taken into account. The interband exchange splits each fourfold degenerate exciton state, in $C_{2 \mathrm{v}}$ symmetry, into four states consisting of a dark state (pure triplet), two states optically excited by $x$ - and $y$-polarized 
light, and one state excited only by $z$-polarized light. The $x-y$ polarized states, observed in our setup, are given by

$$
\left|x_{ \pm}\right\rangle=\left|h_{1}\right\rangle\left|e_{2}\right\rangle \pm i\left|h_{2}\right\rangle\left|e_{1}\right\rangle \text {. }
$$

As may be easily verified, the states $x_{+}$and $x_{-}$are fully polarized along the [110] and [1 $\overline{1} 0]$ directions, respectively. It can be shown that $\left|x_{+}\right\rangle$and $\left|x_{-}\right\rangle$are separated in energy by $\Delta E \propto \sin 2 \phi$. The constant of proportionality is of the order of the exchange energy that in bulk InP is less than 100 $\mu \mathrm{eV}$, but would be enhanced in SAD's because of the strong confinement along the growth direction, as is seen in quantum wells. The observed energy splitting suggests a reasonable exchange energy of the order of $1 \mathrm{meV}$. A more accurate quantitative analysis that takes into account the detailed structure of the envelope wave functions is made difficult by the complicated nature of the hole confinement potential in InP/Ga $\mathrm{Ga}_{0.5} \mathrm{In}_{0.5} \mathrm{P}$ systems. ${ }^{4}$ The difference in oscillator strength between the constituents of a doublet, $\Delta f$, is also proportional to $\sin 2 \phi$ and thus to $\Delta E$. This is qualitatively in agreement with the observed spectrum. ${ }^{15}$ It is important to note that the energy splitting between the [110] and [1 10$]$ polarized exciton emissions is caused by the combined effect of the exchange interaction and the asymmetric strain; the latter causes the symmetry reduction. This effect is similar to the stress exchange interplay observed in bulk semiconductors. ${ }^{16}$

We found that the observed splitting energy differs from dot to dot. For example, even if the PL is observed at 1.870 $\mathrm{eV}$ as a single peak in Fig. 3(d), it is composed of two constituents and they are selected by using a polarizer [see Figs. 3(e) and 3(f)]. Such a fine splitting resolved by polarization is also observed at the lower energy edge of the PL band $(\sim 1.64 \mathrm{eV})$. On the other hand, the polarization-dependent energy shifts of some PL peaks marked by open and closed triangles were not observed within our resolution limit. As mentioned before, the actual magnitude of the splitting would depend on the electron-hole envelope functions and hence on the size and strain distribution of each SAD. The observed dispersion in the energy splitting, therefore, implies that the structure of the SAD's is affected by the long range inhomogeneities in the composition modulated $\mathrm{Ga}_{0.5} \operatorname{In}_{0.5} \mathrm{P}$ matrix. It may be noted that a fine splitting of the exciton level is also observed in the PL and the PL excitation spectra of excitons trapped by potential minimum induced by the fluctuation of the width of a quantum well. ${ }^{17}$

In summary, we observed optical anisotropy in the PL spectra of the InP SAD grown in $\mathrm{Ga}_{0.5} \mathrm{In}_{0.5} \mathrm{P}$. The optical anisotropy is observed in the PL bands of both the InP SAD's and the $\mathrm{Ga}_{0.5} \mathrm{In}_{0.5} \mathrm{P}$ matrix. By comparing the $\mathrm{PL}$ spectra of the samples grown at different temperatures, we found that the optical anisotropy is not related to the shape of the SAD's, but results from the anisotropy in the microscopic structure of the SAD's. This is consistent with the expected symmetry reduction of a cubic unit cell under lateral stress. In the $\mu$-PL spectra we observed a fine splitting (doublet structure) of the exciton states in single quantum dots. This fine structure arises from the electron-hole exchange interaction in the presence of structural asymmetry. It is demonstrated that each peak in the doublet can be selected by a polarizer set inside the microscope. The energy separations of the doublet lines differ from dot to dot. This suggests that the anisotropy of the confined exciton states is influenced by the structure of the matrix.
*Electronic address: mitsuru@sqdp.trc-net.co.jp

${ }^{1}$ P. M. Petroff and S. P. DenBaars, Superlattices Microstruct. 15, 15 (1994), and references therein.

${ }^{2}$ K. Georgsson, N. Carlsson, L. Samuelson, W. Seifert, and L. R. Wallenberg, Appl. Phys. Lett. 67, 2981 (1995).

${ }^{3}$ L. Samuelson, N. Carlsson, P. Castrillo, A. Gustafsson, D. Hessman, J. Lindahl, L. Montelius, A. Petersson, M.-E. Pistol, and W. Seifert, Jpn. J. Appl. Phys., Part 1 34, 4392 (1995).

${ }^{4}$ C. Pryor, M.-E. Pistol, and L. Samuelson, Phys. Rev. B 56, 10404 (1997).

${ }^{5}$ J.-Y. Marzin and G. Bastard, Solid State Commun. 92, 437 (1994).

${ }^{6}$ M. Grundmann, O. Stier, and D. Bimberg, Phys. Rev. B 52, 11969 (1995).

${ }^{7}$ A. Wojs, P. Hawrylak, S. Fafard, and L. Jacak, Phys. Rev. B 54, 5604 (1996).

${ }^{8}$ H.-W. Ren, M. Sugisaki, S. Sugou, K. Nishi, A. Gomyo, and Y. Masumoto, Jpn. J. Appl. Phys., Part 1 (to be published April 1999).

${ }^{9}$ M. Sugisaki, H.-W. Ren, S. Sugou, K. Nishi, and Y. Masumoto, Solid-State Electron. 42, 1325 (1998); M. Sugisaki, H.-W. Ren, S. Sugou, K. Nishi, T. Okuno, and Y. Masumoto, Physica B 256-258, 169 (1998).
${ }^{10}$ S.-H. Wei, A. Franceschetti, and A. Zunger, in Optoelectronic Materials: Ordering, Composition Modulation, and SelfAssembled Structures, edited by E. D. Jones, A. Mascarenhas, and P. Petroff, MRS Symposia Proceedings No. 417 (Materials Research Society, Pittsburgh, 1996), p. 3.

${ }^{11}$ H. Saito, K. Nishi, S. Sugou, and Y. Sugimoto, Appl. Phys. Lett. 71, 590 (1997).

${ }^{12}$ Y. Nabetani, T. Ishikawa, S. Noda, and A. Sasaki, J. Appl. Phys. 76, 347 (1994).

${ }^{13}$ M. Henini, S. Sanguinetti, S. C. Fortina, E. Grilli, M. Guzzi, G. Panzarini, L. C. Andreani, M. D. Upward, P. Moriarty, P. H. Beton, and L. Eaves, Phys. Rev. B 57, 6815 (1998).

${ }^{14}$ Y. Zhang and A. Mascarenhas, Phys. Rev. B 57, 12245 (1998).

${ }^{15}$ The doublet that has large $\Delta E$ tends to show large differences in the PL intensities between the constituents, which reflects $\Delta f$. Details will be reported elsewhere.

${ }^{16}$ See, for example, Excitons, edited by K. Cho (Springer-Verlag, Berlin, 1979).

${ }^{17}$ D. Gammon, E. S. Snow, and D. S. Katzer, Appl. Phys. Lett. 67, 2391 (1995); D. Gammon, E. S. Snow, B. V. Shanabrook, D. S. Katzer, and D. Park, Science 273, 87 (1996); Phys. Rev. Lett. 76, 3005 (1996). 